\title{
On SA, CA, and GA numbers
}

\author{
Geoffrey Caveney \\ 7455 North Greenview \#426, Chicago, IL 60626, USA \\ E-mail: rokirovka@gmail.com \\ Jean-Louis Nicolas \\ Université de Lyon; CNRS; Université Lyon 1; \\ Institut Camille Jordan, Mathématiques, \\ 21 Avenue Claude Bernard, F-69622 Villeurbanne cedex, France \\ E-mail: nicolas@math.univ-lyon1.fr \\ Jonathan Sondow \\ 209 West 97th Street \#6F, New York, NY 10025, USA \\ E-mail: jsondow@alumni.princeton.edu \\ Tel.: +1-646-306-1909
}

\begin{abstract}
Gronwall's function $G$ is defined for $n>1$ by $G(n)=\frac{\sigma(n)}{n \log \log n}$ where $\sigma(n)$ is the sum of the divisors of $n$. We call an integer $N>1$ a GA1 number if $N$ is composite and $G(N) \geq G(N / p)$ for all prime factors $p$ of $N$. We say that $N$ is a GA2 number if $G(N) \geq G(a N)$ for all multiples $a N$ of $N$. In arXiv 1110.5078, we used Robin's and Gronwall's theorems on $G$ to prove that the Riemann Hypothesis $(\mathrm{RH})$ is true if and only if 4 is the only number that is both GA1 and GA2. Here, we study GA1 numbers and GA2 numbers separately. We compare them with superabundant (SA) and colossally abundant (CA) numbers (first studied by Ramanujan). We give algorithms for computing GA1 numbers; the smallest one with more than two prime factors is 183783600 , while the smallest odd one is 1058462574572984015114271643676625 . We find nineteen GA2 numbers $\leq 5040$, and prove that a GA2 number $N>5040$ exists if and only if $\mathrm{RH}$ is false, in which case $N$ is even and $>10^{8576}$.
\end{abstract}

Keywords Colossally abundant · Riemann Hypothesis · Robin's inequality - sum-of-divisors function · superabundant

Mathematics Subject Classification (2000) 11M26 $11 \mathrm{~A} 41 \cdot 11 \mathrm{Y} 55$ 


\section{Introduction}

The sum-of-divisors function $\sigma$ is defined by

$$
\sigma(n):=\sum_{d \mid n} d
$$

For example, $\sigma(4)=7$.

In 1913, Gronwall [7] found the maximal order of $\sigma$.

Theorem 1 (Gronwall) The function

$$
G(n):=\frac{\sigma(n)}{n \log \log n} \quad(n>1)
$$

satisfies

$$
\limsup _{n \rightarrow \infty} G(n)=e^{\gamma}=1.78107 \ldots,
$$

where $\gamma$ is the Euler-Mascheroni constant.

In 1915, Ramanujan proved an asymptotic inequality for Gronwall's function $G$, assuming the Riemann Hypothesis $(\mathrm{RH})$. Ramanujan's result was shown in the second part of his thesis. The first part was published in 1915 [12] while the second part was not published until much later, in 1997 [13].

Theorem 2 (Ramanujan) If the Riemann Hypothesis is true, then

$$
G(n)<e^{\gamma} \quad(n \gg 1) .
$$

Here, $n \gg 1$ means for all sufficiently large $n$.

In 1984, without being aware of Ramanujan's theorem, Robin [14] proved that a stronger statement about the function $G$ is equivalent to RH.

Theorem 3 (Robin) The Riemann Hypothesis is true if and only if

$$
G(n)<e^{\gamma} \quad(n>5040) .
$$

The condition (11) is called Robin's inequality. Table 1 gives the twentysix known numbers $r$ for which the reverse inequality $G(r) \geq e^{\gamma}$ holds (see [17, Sequence A067698]), together with the value of $G(r)$ (truncated). (The " $a(r)$ " column is explained in $\$ 4$, and the " $Q(r)$ " column in 97.1 ,

In [14] Robin also proved, unconditionally, that

$$
G(n) \leq e^{\gamma}+\frac{0.6482 \ldots}{(\log \log n)^{2}} \quad(n>1)
$$




\begin{tabular}{|c|c|c|c|c|c|c|c|c|c|}
\hline$r$ & $\overline{\mathrm{SA}}$ & $\overline{\mathrm{CA}}$ & $\overline{\mathrm{GA1}}$ & $\overline{\mathrm{GA2}}$ & Factorization & $\sigma(r) / r$ & $G(r)$ & $a(r)$ & $Q(r)$ \\
\hline 3 & & & & $\checkmark$ & 3 & 1.333 & 14.177 & 0 & \\
\hline 4 & $\checkmark$ & & $\checkmark$ & $\checkmark$ & $2^{2}$ & 1.750 & 5.357 & 0 & -0.763 \\
\hline 5 & & & & $\checkmark$ & 5 & 1.200 & 2.521 & 0 & \\
\hline 6 & $\checkmark$ & $\checkmark$ & & $\checkmark$ & $2 \cdot 3$ & 2.000 & 3.429 & 0 & 4.134 \\
\hline 8 & & & & $\checkmark$ & $2^{3}$ & 1.875 & 2.561 & 0 & 2.091 \\
\hline 9 & & & & & $3^{2}$ & 1.444 & 1.834 & 4 & 7.726 \\
\hline 10 & & & & $\checkmark$ & $2 \cdot 5$ & 1.800 & 2.158 & 0 & 1.168 \\
\hline 12 & $\checkmark$ & $\checkmark$ & & $\checkmark$ & $2^{2} \cdot 3$ & 2.333 & 2.563 & 0 & 2.090 \\
\hline 16 & & & & & $2^{4}$ & 1.937 & 1.899 & 3 & 1.348 \\
\hline 18 & & & & $\checkmark$ & $2 \cdot 3^{2}$ & 2.166 & 2.041 & 0 & 1.679 \\
\hline 20 & & & & & $2^{2} \cdot 5$ & 2.100 & 1.913 & 3 & 2.799 \\
\hline 24 & $\checkmark$ & & & $\checkmark$ & $2^{3} \cdot 3$ & 2.500 & 2.162 & 0 & 1.185 \\
\hline 30 & & & & & $2 \cdot 3 \cdot 5$ & 2.400 & 1.960 & 2 & 1.749 \\
\hline 36 & $\checkmark$ & & & $\checkmark$ & $2^{2} \cdot 3^{2}$ & 2.527 & 1.980 & 0 & 1.294 \\
\hline 48 & $\checkmark$ & & & $\checkmark$ & $2^{4} \cdot 3$ & 2.583 & 1.908 & 0 & 1.132 \\
\hline 60 & $\checkmark$ & $\checkmark$ & & $\checkmark$ & $2^{2} \cdot 3 \cdot 5$ & 2.800 & 1.986 & 0 & 1.290 \\
\hline 72 & & & & $\checkmark$ & $2^{3} \cdot 3^{2}$ & 2.708 & 1.863 & 0 & 1.160 \\
\hline 84 & & & & & $2^{2} \cdot 3 \cdot 7$ & 2.666 & 1.791 & 10 & 1.430 \\
\hline 120 & $\checkmark$ & $\checkmark$ & & $\checkmark$ & $2^{3} \cdot 3 \cdot 5$ & 3.000 & 1.915 & 0 & 1.128 \\
\hline 180 & $\checkmark$ & & & $\checkmark$ & $2^{2} \cdot 3^{2} \cdot 5$ & 3.033 & 1.841 & 0 & 1.078 \\
\hline 240 & $\checkmark$ & & & $\checkmark$ & $2^{4} \cdot 3 \cdot 5$ & 3.100 & 1.822 & 0 & 1.051 \\
\hline 360 & $\checkmark$ & $\checkmark$ & & $\checkmark$ & $2^{3} \cdot 3^{2} \cdot 5$ & 3.250 & 1.833 & 0 & 1.044 \\
\hline 720 & $\checkmark$ & & & & $2^{4} \cdot 3^{2} \cdot 5$ & 3.358 & 1.782 & 7 & 1.028 \\
\hline 840 & $\checkmark$ & & & & $2^{3} \cdot 3 \cdot 5 \cdot 7$ & 3.428 & 1.797 & 3 & 1.065 \\
\hline 2520 & $\checkmark$ & $\checkmark$ & & $\checkmark$ & $2^{3} \cdot 3^{2} \cdot 5 \cdot 7$ & 3.714 & 1.804 & 0 & 1.015 \\
\hline 5040 & $\checkmark$ & $\checkmark$ & & $\checkmark$ & $2^{4} \cdot 3^{2} \cdot 5 \cdot 7$ & 3.838 & 1.790 & 0 & 1.007 \\
\hline
\end{tabular}

TABLE 1 - The set $\mathcal{R}=\left\{r \leq 5040: G(r) \geq e^{\gamma}=1.781 \ldots\right\}$, which contains the subset $\mathcal{R}_{2}=\{N \leq 5040: N$ is GA2 $\}$. 
with equality for $n=12$. This refines the inequality $\lim \sup _{n \rightarrow \infty} G(n) \leq e^{\gamma}$ from Gronwall's theorem.

Recently, the authors [3] used Robin's results to derive another reformulation of RH. Before recalling its statement, we give three definitions and an example.

A positive integer $N$ is a GA1 number if $N$ is composite and the inequality

$$
G(N) \geq G(N / p)
$$

holds for all prime factors $p$ of $N$. The first few GA1 numbers are

$$
N=4,14,22,26,34,38,46,58,62,74,82,86,94,106,118,122,134,142, \ldots
$$

(see [17, Sequence A197638]), and (see \$5.3) the smallest odd GA1 number is

$$
N=1058462574572984015114271643676625 .
$$

An integer $N>1$ is a GA2 number if

$$
G(N) \geq G(a N)
$$

for all multiples $a N$ of $N$. The nineteen known GA2 numbers (see Theorem 5 and [17, Sequence A197369]) are

$$
N=3,4,5,6,8,10,12,18,24,36,48,60,72,120,180,240,360,2520,5040 .
$$

Every GA2 number $>5$ is even. (Proof. If $N$ is odd, then $\sigma(2 N)=3 \sigma(N)$, and if $N$ is also GA2, we get

$$
\frac{3}{2} \leq \frac{3 G(N)}{2 G(2 N)}=\frac{\log \log 2 N}{\log \log N}
$$

which implies $N<7$.)

Finally, a composite number is extraordinary if it is both GA1 and GA2.

For example, the smallest extraordinary number is 4 . To see this, we first compute $G(4)=5.357 \ldots$. Then, as $G(2)<0$, it follows that 4 is a GA1 number. Since Robin's unconditional bound (2) implies

$$
G(n)<e^{\gamma}+\frac{0.6483}{(\log \log 5)^{2}}=4.643 \ldots<G(4) \quad(n \geq 5),
$$

we get that 4 is also GA2. Thus 4 is an extraordinary number.

We can now recall our results from [3, Theorem 6 and Corollary 8]. 
Theorem 4 (Caveney-Nicolas-Sondow) (i). The Riemann Hypothesis is true if and only if 4 is the only extraordinary number.

(ii). If there is any counterexample to Robin's inequality, then the maximum $\mu:=\max \{G(n): n>5040\}$ exists and the least number $N>5040$ with $G(N)=\mu$ is extraordinary.

If there exists an extraordinary number $N>4$, then $N$ is even (as 5 is not GA1, and no GA2 number $>5$ is odd) and $N>10^{8576}$ (since no GA1 number lies in the interval $[5,5040]$, and no GA2 number lies in $\left[5041,10^{8576}\right]$ - see Corollary 1).

In the present paper, we study GA1 numbers and GA2 numbers separately.

Preliminary facts about GA1 numbers and GA2 numbers were given in [3]. We recall two of them and make a definition.

Fact 1 (proved by elementary methods in [3, §5]). The GA1 numbers with exactly two (not necessarily distinct) prime factors are precisely 4 and $2 p$, for primes $p \geq 7$.

We call such GA1 numbers improper, while GA1 numbers with at least three (not necessarily distinct) prime factors will be called proper.

The smallest proper GA1 number is $\nu:=183783600$ (see $\$ 5.3$ and [17, Sequence A201557]). The number $\nu$ was mentioned in [3, equation (3)] as an example of a (proper) GA1 number that is not a GA2 number (because $G(\nu)<G(19 \nu))$.

Fact 2 (see [3, Lemma 10]). If $n_{0}$ is a positive integer, then

$$
\limsup _{a \rightarrow \infty} G\left(a n_{0}\right)=e^{\gamma},
$$

which yields the implication

$$
N \text { is } G \text { A2 } \Longrightarrow G(N) \geq e^{\gamma} \text {. }
$$

An application is an alternate proof that any GA2 number $N>5$ is even. Namely, as 7 and 9 are not GA2, and as Theorem 2 in [4] says that an integer $n>9$ is even if $G(n) \geq e^{\gamma}$, the result follows from (3).

By the method of [3, §5], one can prove two additional properties of GA1 numbers.

Fact 3. The only prime power GA1 number $N=p^{k}$ is $N=4$.

Fact 4. A product of three distinct primes $p_{1} p_{2} p_{3}$ cannot be a GA1 number. (See $\$ 6.2$ for a more general result proved by other methods.) 
The rest of the paper is organized as follows. The next subsection establishes notation. In $\$ 2$ we recall the definitions of superabundant (SA) and colossally abundant (CA) numbers and review some of their properties. In $\$ 3$ we prove six lemmas needed later. In $\$ 4$ we give an analog of Theorem 4 for GA2 numbers; in particular, if $R H$ is false, then infinitely many GA2 numbers exist, and any number $N>5040$ for which $G(N)=\max \{G(n): n>5040\}$ is both GA2 and $C A$. In the final four sections we study proper GA1 numbers : $\$ 5$ compares them with SA and CA numbers, $\$ 6$ is concerned with their prime factors, $\$ 7$ gives algorithms for computing them, and $\$ 8$ estimates the number of them up to $x$.

\subsection{Notation}

We let $p$ always denote a prime.

Let $v_{p}(n)$ denote the exponent on $p$ in the prime factorization

$$
n=\prod_{p} p^{v_{p}(n)}
$$

For $n \geq 1$, we denote the number of prime factors of $n$ counted with multiplicity by

$$
\Omega(n):=\sum_{p} v_{p}(n)
$$

For $n>1$, we denote the largest prime factor of $n$ by

$$
P(n):=\max \{p: p \mid n\}=\max \left\{p: v_{p}(n)>0\right\} .
$$

As usual, Chebychev's function is defined as

$$
\theta(x):=\sum_{p \leq x} \log p .
$$

\section{Review of properties of SA and CA numbers}

Superabundant and colossally abundant numbers were first introduced by Ramanujan, who called them generalized highly composite and generalized super highly composite numbers, respectively (cf. [13, §59]). They were rediscovered later by Alaoglu and Erdôs [1].

A superabundant $(S A)$ number is a positive integer $N$ such that

$$
\frac{\sigma(N)}{N}>\frac{\sigma(n)}{n} \quad(0<n<N)
$$


The first few SA numbers are (see [17, Sequence A004394])

$$
N=1,2,4,6,12,24,36,48,60,120,180,240,360,720,840,1260,1680, \ldots .
$$

A colossally abundant ( $C A$ ) number is a positive integer $N$ for which there exists an exponent $\varepsilon>0$ such that

$$
\frac{\sigma(N)}{N^{1+\varepsilon}} \geq \frac{\sigma(n)}{n^{1+\varepsilon}} \quad(n>1) .
$$

Such an exponent $\varepsilon$ is called a parameter of $N$. The sequence of CA numbers (compare [17, Sequence A004490]) begins

$N=1,2,6,12,60,120,360,2520,5040,55440,720720,1441440,4324320, \ldots$

From (44), it is easy to show that every $C A$ number is also $S A$.

Now let $N$ denote an SA or CA number. Then (see [1, Theorems 1 and 3] or [13, §59])

$$
N=2^{k_{2}} \cdot 3^{k_{3}} \cdot 5^{k_{5}} \cdots p^{k_{p}} \quad \Longrightarrow \quad k_{2} \geq k_{3} \geq k_{5} \geq \cdots \geq k_{p}
$$

with $k_{p}=1$ unless $N=4$ or 36 , and [1, Theorem 7]

$$
p=P(N) \sim \log N \quad(N \rightarrow \infty) .
$$

We recall some properties of CA numbers (see [1, 2, 3, 6, 9, 13, 14, 15]).

Note first that for any fixed positive integer $k$, the quantity

$$
F(t, k):=\frac{\log \left(1+\frac{1}{t+t^{2}+\cdots+t^{k}}\right)}{\log t}
$$

is decreasing on the interval $1<t<\infty$, and the function $t \mapsto F(t, k)$ maps the interval onto the positive real numbers. Hence, given $\varepsilon>0$, we may define $x_{k}=x_{k}(\varepsilon)>1$ by

$$
F\left(x_{k}, k\right)=\varepsilon .
$$

(See [14, p. 189] and [13, §61 and §69].) In particular, when $k=1$ we set $x=x_{1}=x_{1}(\varepsilon)$, so that

$$
F(x, 1)=F\left(x_{1}, 1\right)=\frac{\log \left(1+\frac{1}{x}\right)}{\log x}=\varepsilon .
$$


It is convenient to set $x_{0}=+\infty$. From the decreasingness of $F(t, k)$ with respect to both $t$ and $k$, it follows that the sequence $\left(x_{k}\right)_{k \geq 0}$ is decreasing.

If $N$ is a CA number of parameter $\varepsilon$ and $p$ divides $N$ with $v_{p}(N)=k$, then applying (44) with $n=N p$ yields

$$
\varepsilon \geq F(p, k+1) \quad \text { i.e. } \quad p \geq x_{k+1}
$$

while, if $k>0$, applying (4) with $n=N / p$ yields

$$
\varepsilon \leq F(p, k) \quad \text { i.e. } \quad p \leq x_{k} .
$$

Let $K$ be the largest integer such that $x_{K} \geq 2$. Then from (9), for all $p$ 's we have $2 \leq p \leq x_{k}$ and

$$
k=v_{p}(N) \leq K .
$$

Now define the set

$$
\mathcal{E}:=\{F(p, k): p \text { is prime and } k \geq 1\}
$$

Its largest element is

$$
\max \mathcal{E}=F(2,1)=\frac{\log (3 / 2)}{\log 2}=0.5849 \ldots,
$$

and its infimum is

$$
\inf \mathcal{E}=\lim _{k \rightarrow \infty} F(p, k)=0
$$

for any fixed prime $p$.

If $\varepsilon \notin \mathcal{E}$, then no $x_{k}$ is a prime number and there exists a unique CA number $N=N(\varepsilon)$ of parameter $\varepsilon$; moreover, $N$ is given by either of the equivalent formulas

$$
N=\prod_{p<x} p^{k_{p}} \quad \text { with } x_{k_{p}+1}<p<x_{k_{p}}
$$

or

$$
N=\prod_{k=1}^{K} \prod_{p<x_{k}} p
$$

In particular, if $\varepsilon>\max \mathcal{E}$, then $x=x_{1}<2, K=0$ and $N(\varepsilon)=1$.

If $\varepsilon \in \mathcal{E}$, then some $x_{k}$ is prime, and it is highly probable that only one $x_{k}$ is prime. But (see [6, Proposition 4]), from the theorem of six exponentials it is only possible to show that at most two $x_{k}$ 's are prime. (Compare [9, 
p. 538].) Therefore there are either two or four CA numbers of parameter $\varepsilon$, defined by

$$
N=\prod_{k=1}^{K} \prod_{\substack{p \leq x_{k} \\ \text { or } \\ p<x_{k}}} p
$$

Here, if $x_{k}$ is a prime $p$ for some $k$, then $p$ may or may not be a factor in the inner product. (This can occur for at most two values of $k$.) In other words, if $x_{k-1}<p<x_{k}$, then the exponent $v_{p}(N)$ of $p$ in $N$ is $k$, while if $p=x_{k}$, the exponent may be $k$ or $k-1$. In particular, if $N$ is the largest CA number of parameter $\varepsilon$, then

$$
F(p, 1)=\varepsilon \quad \Longrightarrow \quad P(N)=p .
$$

Note that, since if $\varepsilon \notin \mathcal{E}$, then $x_{k}$ is not prime, formula (10) gives the same value as (11). Therefore, for any $\varepsilon$, formula (11) gives all the possible values of a CA number $N$ of parameter $\varepsilon$. (Thus $N$ is a product of "primorials" [17, Sequence A002110].)

\section{Six lemmas}

The case $k=2$ of the following lemma was proved in [14, p. 190].

Lemma 1 For $k \geq 2$, we have the upper bound

$$
x_{k}<(k x)^{1 / k} .
$$

Proof. Since the function $t \mapsto F(t, k)$ is strictly decreasing on $1<t<\infty$, to prove $x_{k}<z:=(k x)^{1 / k}$, it suffices to show $F(z, k)<F\left(x_{k}, k\right)$. As (7) and (8) imply $F\left(x_{k}, k\right)=\varepsilon=F(x, 1)$, this reduces to showing $F(z, k)<F(x, 1)$.

Since $z>1$ and $k \geq 2$, we have

$$
\begin{aligned}
F(z, k) & =\log \left(1+\frac{1}{z+z^{2}+\cdots+z^{k}}\right) \frac{1}{\log z} \\
& <\frac{1}{\left(z+z^{2}+\cdots+z^{k}\right) \log z}=\frac{k}{\left(z+z^{2}+\cdots+z^{k}\right) \log k x} \\
& <\frac{k}{\left(k-1+z^{k}\right) \log x} \leq \frac{k}{\left(\frac{k}{2}+k x\right) \log x}=\frac{1}{\left(x+\frac{1}{2}\right) \log x} \\
& <\log \left(1+\frac{1}{x}\right) \frac{1}{\log x}=F(x, 1),
\end{aligned}
$$


using the lower bound $\log \left(1+\frac{1}{t}\right)>\left(t+\frac{1}{2}\right)^{-1}$, valid for $t>0$. This proves the desired inequality.

In the proof of Theorem 5 (iii), we will need the following result (see [11, Lemma 4]).

Lemma 2 Given a $C A$ number $N_{0}$ of parameter $\varepsilon_{0}$, let $N>N_{0}$ be a number satisfying

$$
n \geq N_{0} \quad \Longrightarrow \quad \frac{\sigma(n)}{n^{1+\varepsilon}} \leq \frac{\sigma(N)}{N^{1+\varepsilon}}
$$

for some fixed $\varepsilon>0$. Then $N$ is $C A$ of parameter $\varepsilon$.

Proof. Since $N_{0}$ is CA of parameter $\varepsilon_{0}$, we have

$$
\frac{\sigma(N)}{\sigma\left(N_{0}\right)} \leq\left(\frac{N}{N_{0}}\right)^{1+\varepsilon_{0}}
$$

On the other hand, (13) yields

$$
\frac{\sigma(N)}{\sigma\left(N_{0}\right)} \geq\left(\frac{N}{N_{0}}\right)^{1+\varepsilon}
$$

Hence $\varepsilon \leq \varepsilon_{0}$.

In view of (13), to prove that $N$ is $\mathrm{CA}$ of parameter $\varepsilon$, we only need to show that

$$
n<N_{0} \quad \Longrightarrow \quad \frac{\sigma(n)}{n^{1+\varepsilon}} \leq \frac{\sigma(N)}{N^{1+\varepsilon}} .
$$

If $n<N_{0}$, then since $N_{0}$ is CA and (13) holds, we have

$$
\frac{\sigma(n)}{n^{1+\varepsilon}}=\frac{\sigma(n) n^{\varepsilon_{0}-\varepsilon}}{n^{1+\varepsilon_{0}}} \leq \frac{\sigma\left(N_{0}\right) n^{\varepsilon_{0}-\varepsilon}}{N_{0}^{1+\varepsilon_{0}}} \leq \frac{\sigma\left(N_{0}\right) N_{0}^{\varepsilon_{0}-\varepsilon}}{N_{0}^{1+\varepsilon_{0}}}=\frac{\sigma\left(N_{0}\right)}{N_{0}^{1+\varepsilon}} \leq \frac{\sigma(N)}{N^{1+\varepsilon}} .
$$

This completes the proof of Lemma 2 .

The next lemma provides an estimate for a CA number of parameter $\varepsilon$.

Lemma 3 Let $N$ be a $C A$ number of parameter $\varepsilon<F(2,1)=\log (3 / 2) / \log 2$ and define $x=x(\varepsilon)$ by (8) .

(i). Then

$$
\log N \leq(x)+c \sqrt{x}
$$

for some constant $c>0$. 
(ii). Moreover, if $N$ is the largest $C A$ number 1 of parameter $\varepsilon$, then

$$
(x) \leq \log N \leq(x)+c \sqrt{x} .
$$

Proof. (i). It follows from formula (11) for $N$ that if $x_{k}$ is defined by (17), then

$$
\log N \leq\left(x_{1}\right)+\left(x_{2}\right)+\cdots+\left(x_{K}\right),
$$

where $K$ is the largest integer such that $x_{K} \geq 2$. (Note that $v_{2}(N)=K$ or $K-1$, and that $\varepsilon<F(2,1)$ implies $x>2$ and $K \geq 1$.)

As $t \mapsto F(t, k)$ is decreasing and (17) holds, we have

$$
F(2, K) \geq F\left(x_{K}, K\right)=\varepsilon=F\left(x_{K+1}, K+1\right)>F(2, K+1) .
$$

On the other hand,

$$
F(2, K)=\log \left(1+\frac{1}{2^{K+1}-2}\right) \frac{1}{\log 2}<\frac{1}{\left(2^{K+1}-2\right) \log 2} \leq \frac{1}{2^{K} \log 2}<\frac{2}{2^{K}}
$$

and, from (8),

$$
\varepsilon=\frac{\log \left(1+\frac{1}{x}\right)}{\log x}>\frac{1}{(x+1) \log x} \geq \frac{1}{(x+1)(x-1)}>\frac{1}{x^{2}} .
$$

Thus

implying

$$
\frac{2}{2^{K}}>F(2, K) \geq \varepsilon>\frac{1}{x^{2}}
$$

$$
K<1+\frac{2}{\log 2} \log x
$$

Since $k \mapsto x_{k}$ is decreasing, from (14) we have (compare [13, equation $(368)])$

$$
\log N \leq\left(x_{1}\right)+\left(x_{2}\right)+K\left(x_{3}\right) .
$$

Using $x_{2} \leq \sqrt{2 x}$ and $x_{3} \leq \sqrt[3]{3 x}$ (from Lemma 1), together with (15) and the Prime Number Theorem in the form $(t) \sim t$, we deduce (i).

(ii). From (11), the largest CA number of parameter $\varepsilon$ is

$$
N=\prod_{k=1}^{K} \prod_{p \leq x_{k}} p
$$

1. Note that Ramanujan's definition of CA number of parameter $\varepsilon$ in [13] is not exactly the same as that of Robin in [14, pp. 189-190]. Ramanujan's definition corresponds to the largest CA number of parameter $\varepsilon$ for Robin. 
which implies $(x) \leq \log N$, and (ii) follows from (i).

In the next lemma, we recall the oscillations of Chebychev's function studied by Littlewood.

Lemma 4 There exists a constant $c>0$ such that for infinitely many primes $p$ we have

$$
\theta(p)<p-c \sqrt{p} \log \log \log p,
$$

and for infinitely many other primes $p$ we have

$$
\theta(p)>p+c \sqrt{p} \log \log \log p .
$$

Proof. From Littlewood's theorem (see [10]), we know that there exists a constant $c^{\prime}>0$ such that for a sequence of values of $x$ going to infinity we have

$$
\theta(x)<x-c^{\prime} \sqrt{x} \log \log \log x,
$$

and for a sequence of values of $x^{\prime}$ going to infinity we have

$$
x^{\prime}+c^{\prime} \sqrt{x^{\prime}} \log \log \log x^{\prime}<\theta\left(x^{\prime}\right) .
$$

Let us suppose first that $x$ is large enough and satisfies (18). If $x=p$ is prime, then (18) implies (16). Now assume $x$ is not prime, and let $p$ be the prime following $x$. As the function $t \mapsto t-c \sqrt{t} \log \log \log t$ is increasing, we get

$$
\begin{aligned}
\theta(p)=\theta(x)+\log p & <x-c^{\prime} \sqrt{x} \log \log \log x+\log p \\
& <p-c^{\prime} \sqrt{p} \log \log \log p+\log p,
\end{aligned}
$$

which implies (16) with $c<c^{\prime}$ for $x$ large enough.

The proof of (17) is easier. Let $x^{\prime}$ satisfy (19) and choose the largest prime $p \leq x^{\prime}$. For $c \leq c^{\prime}$, we have

$$
\theta(p)=\theta\left(x^{\prime}\right)>x^{\prime}+c^{\prime} \sqrt{x^{\prime}} \log \log \log x^{\prime}>p+c \sqrt{p} \log \log \log p,
$$

which proves (17).

Lemma 5 Chebychev's function $(x)$ satisfies

$$
(x) \leq(1+\alpha) x,
$$

where

$$
\alpha=\alpha(x):= \begin{cases}0 & \text { if } x \leq 8 \cdot 10^{11} \\ \frac{1}{36260}<0.000028 & \text { otherwise. }\end{cases}
$$


Proof. Schoenfeld (cf. [16, p. 360]) proved $(x) \leq 1.000081 x$ for all $x$, and he mentioned that Brent had checked that $(x)<x$ for $x<10^{11}$. The stronger results stated here are due to Dusart - see [5, p. 2 and Table 6.6].

Lemma 6 Let $\varepsilon$ be a positive real number. For $t>e$, let us set

$$
g(t)=g_{\varepsilon}(t):=\varepsilon \log t-\log \log \log t .
$$

Then there exists a unique real number $t_{0}=t_{0}(\varepsilon)>e$ such that

$$
\frac{1}{\log t_{0} \log \log t_{0}}=\varepsilon
$$

Moreover, $g(t)$ is decreasing for $e<t<t_{0}$ and increasing for $t>t_{0}$.

Proof. The derivative of $g$ is

$$
g^{\prime}(t)=\frac{1}{t}\left(\varepsilon-\frac{1}{\log t \log \log t}\right) .
$$

For $t>e$, both $\log t$ and $\log \log t$ are positive and increasing, and the function $t \mapsto 1 /(\log t \log \log t)$ is a decreasing bijection from $(e,+\infty)$ onto $(0,+\infty)$. Therefore, one can define $t_{0}>e$ by (21).

Then we have $g^{\prime}(t)<0$ for $e<t<t_{0}$, and $g^{\prime}(t)>0$ for $t>t_{0}$, which completes the proof of Lemma 6 .

\section{GA2 numbers}

We first study GA2 numbers. Compare the following result on them with Theorem 4 on extraordinary numbers.

Theorem 5 (i). The set of GA2 numbers $\leq 5040$ is

$\mathcal{R}_{2}:=\{3,4,5,6,8,10,12,18,24,36,48,60,72,120,180,240,360,2520,5040\}$.

(ii). If the Riemann Hypothesis is true, then no GA2 number exceeds 5040.

(iii). If the Riemann Hypothesis is false, then infinitely many GA2 numbers exist; moreover, the inequality

$$
\mu:=\max \{G(n): n>5040\}>e^{\gamma}
$$

holds, and any integer $A>5040$ for which $G(A)=\mu$ is both GA2 and $C A$. 
Proof. (i). Setting

$$
\mathcal{R}^{\prime}:=\{N \leq 5040: N \text { is GA } 2\},
$$

we have to prove that $\mathcal{R}^{\prime}=\mathcal{R}_{2}$.

To show $\mathcal{R}^{\prime} \subset \mathcal{R}_{2}$, choose $N \in \mathcal{R}^{\prime}$. From (3), we have $G(N) \geq e^{\gamma}$, so that

$$
\begin{aligned}
N \in \mathcal{R}:= & \left\{r \leq 5040: G(r) \geq e^{\gamma}\right\} \\
= & \{3,4,5,6,8,9,10,12,16,18,20,24,30,36,48,60,72,84,120,180, \\
& 240,360,720,840,2520,5040\},
\end{aligned}
$$

by calculating the " $r$ " column of Table 1. To show that $N$ belongs to the subset $\mathcal{R}_{2} \subset \mathcal{R}$, define for $r \in \mathcal{R}$ the integer

$$
a(r):= \begin{cases}\min \mathcal{A}_{r}, & \text { if } \mathcal{A}_{r}:=\{a: G(a r)>G(r), \text { ar } \in \mathcal{R}\} \neq \emptyset, \\ 0, & \text { if } \mathcal{A}_{r}=\emptyset .\end{cases}
$$

A computation (see the " $a(r)$ " column of Table 1) shows that

$$
\left\{r \in \mathcal{R}: \mathcal{A}_{r} \neq \emptyset\right\}=\{9,16,20,30,84,720,840\} .
$$

Since $N$ is GA2, it must lie in the complement

$$
\mathcal{R} \backslash\{9,16,20,30,84,720,840\}=\mathcal{R}_{2} .
$$

This shows $\mathcal{R}^{\prime} \subset \mathcal{R}_{2}$.

To prove $\mathcal{R}_{2} \subset \mathcal{R}^{\prime}$, choose $r \in \mathcal{R}_{2}$. To get $r \in \mathcal{R}^{\prime}$, we need to show that $G(r) \geq G(a r)$, for any multiple ar of $r$. We consider two cases.

Case $1:$ ar $\leq$ 5040. If $a r \in \mathcal{R}$, then since $r \in \mathcal{R}_{2}$, relations (23) and (22) imply $G(a r) \leq G(r)$. On the other hand, if $a r \notin \mathcal{R}$, then $G(a r)<e^{\gamma} \leq G(r)$. Thus $G(r) \geq G(a r)$ whenever $a r \leq 5040$.

Before considering Case 2, we recall that in [14, p. 204 (c)] Robin proved that if $C$ is the largest CA number with $P(C)<20000$, then there is no counterexample $\leq C$ to his inequality (11). From the property (5) of CA numbers, we have $\log C \geq(20000)$, where $(x)$ is Chebychev's function.

We also recall that in [16, p. 359, Corollary 2], Schoenfeld proved that

$$
(x)>x-\frac{x}{8 \log x} \quad(x \geq 19421) .
$$

A calculation then gives the inequalities

$$
(20000)>20000-\frac{20000}{8 \log 20000}>19747
$$


which, together with Robin's result on $C$, yield the implication

$$
5040<n<e^{19747} \Longrightarrow G(n)<e^{\gamma} .
$$

Case $2:$ ar $>$ 5040. If $\log a r<19747$, then (24) gives $G(a r)<e^{\gamma} \leq G(r)$. On the other hand, if $\log a r \geq 19747$, then from (2) we get

$$
G(a r)<e^{\gamma}+\frac{0.6483}{(\log 19747)^{2}}=1.787 \ldots<1.790 \ldots=\min _{r^{\prime} \in \mathcal{R}_{2}} G\left(r^{\prime}\right) \leq G(r) .
$$

Thus $G(r) \geq G(a r)$ whenever ar $>5040$.

This shows that, in both Cases 1 and 2, all elements $r$ of $\mathcal{R}_{2}$ are GA2 numbers, so that $\mathcal{R}_{2} \subset \mathcal{R}^{\prime}$. Finally, since we already have $\mathcal{R}_{2} \supset \mathcal{R}^{\prime}$, we get $\mathcal{R}_{2}=\mathcal{R}^{\prime}$. This proves (i).

(ii). If RH holds, then by Robin's theorem there is no number $n>5040$ with $G(n) \geq e^{\gamma}$, while from (3) a GA2 number $N$ must satisfy $G(N) \geq e^{\gamma}$.

(iii). Let us assume that $\mathrm{RH}$ fails. Set

$$
\Theta:=\sup _{\zeta(\rho)=0} \Re(\rho)
$$

so that

$$
1 / 2<\Theta \leq 1
$$

Let $N$ denote a CA number of parameter $\varepsilon$, and define $x=x(\varepsilon)$ by (8). If $p:=P(N)$ and if $p^{+}$is the prime following $p$, then from (11) we have

$$
p \leq x_{1}=x \leq p^{+}
$$

which implies $x \sim p$ as $N \rightarrow \infty$. Further, from (6), we get $p \sim \log N$, which implies

$$
x \sim \log N \quad(N \rightarrow \infty) .
$$

In [15, p. 241], it is proved that as $N \rightarrow \infty$

$$
G(N)=e^{\gamma}\left(1+\Omega_{+}\left(x^{-b}\right)\right) \quad(1-\Theta<b<1 / 2)
$$

which implies that

$$
G(N)=e^{\gamma}\left(1+\Omega_{+}\left((\log N)^{-b}\right)\right) \quad(1-\Theta<b<1 / 2) .
$$

(Here the notation " $f(N)=\Omega_{+}(g(N))$ as $N \rightarrow \infty$ " means that $f(N)>g(N)$ infinitely often, and should not be confused with the notation $\Omega(n)$ in $\$ 1.1$.) 
Therefore, there exist infinitely many CA numbers $N$ satisfying $G(N)>e^{\gamma}$, and, for all $t$, we have $\max _{n \geq t} G(n)>e^{\gamma}$.

Now we construct two sequences $A_{1}, A_{2}, \ldots$ and $A_{1}^{\prime}, A_{2}^{\prime}, \ldots$, as follows. Let $A_{1}$ (resp., $A_{1}^{\prime}$ ) be the smallest (resp., largest) 2 integer $>5040$ such that $G\left(A_{1}\right)=G\left(A_{1}^{\prime}\right)=\mu$.

Given $i \geq 2$, assume that $A_{1}, A_{2}, \ldots, A_{i-1}$ and $A_{1}^{\prime}, A_{2}^{\prime}, \ldots, A_{i-1}^{\prime}$ have been defined. Set $\mu_{i}:=\max _{n>A_{i-1}^{\prime}} G(n)$ and let $A_{i}$ (resp., $A_{i}^{\prime}$ ) be the smallest (resp., largest) integer $>A_{i-1}^{\prime}$ with $G\left(A_{i}\right)=G\left(A_{i}^{\prime}\right)=\mu_{i}$. Since we have $\mu_{i}>e^{\gamma}=\lim \sup G(n)$, infinitely many $A_{i}$ 's can be found. The numbers $A_{i}$ are such that

$$
n>A_{i} \Longrightarrow G(n) \leq G\left(A_{i}\right)
$$

and, therefore, are GA2.

In the same way, $A$ is proved to be GA2, using $A>5040$ and $G(A)=\mu$. To show that $A$ is $\mathrm{CA}$, we apply Lemma 2 with $N_{0}=55040, \varepsilon_{0}=0.03$, $N=A$, and $\varepsilon=1 /(\log A \log \log A)$; since $A$ is GA2 and $A>5040$, from (24) and (3) we obtain that $N=A>e^{19747}>N_{0}$. For $n \geq N_{0}$, from the definition of $A$ we have $G(n) \leq G(A)$. Since $e<N_{0}<A$ holds, it follows from Lemma 6 that, on the interval $\left[N_{0},+\infty\right.$ ), the function $g(t)$ (defined by (20) ) attains its minimum at $t=A$. Thus, for $n \geq N_{0}$, we have

$$
\frac{\sigma(n)}{n^{1+\varepsilon}}=G(n) \frac{\log \log n}{n^{\varepsilon}}=G(n) e^{-g(n)} \leq G(A) e^{-g(n)} \leq G(A) e^{-g(A)}=\frac{\sigma(A)}{A^{1+\varepsilon}}
$$

and so (13) holds. Applying Lemma 2 completes the proof of (iii).

Here is a corollary of the proof of Theorem 5.

Corollary 1 There is no GA2 or extraordinary number between 5041 and $10^{8576}$.

Proof. Since $10^{8576}<e^{19747}$, this follows from (24).

\section{Comparison between CA and GA1 numbers}

In this section, we study GA1 numbers. We begin by comparing them with CA numbers.

2. It is highly probable that $A_{1}=A_{1}^{\prime}$. A difficult question is whether $G$ is injective. 


\subsection{CA and GA1}

By revisiting the proof of [15, Theorem 3, p. 242], we shall prove the following results.

Lemma 7 Let $N$ be a $C A$ number of parameter $\varepsilon>0$ and assume that $p:=P(N) \geq 5$. If

$$
\varepsilon>\frac{1}{\log (N / p) \log \log (N / p)},
$$

then $N$ is also a GA1 number.

Proof. Let $q$ be a prime factor of $N$. It follows from (5) that $6 p$ divides $N$ and that $N / q \geq N / p \geq 6>e$, which implies $\log \log (N / q)>\log \log e=0$. Since $N$ is a CA number, from (4) one has

$$
\frac{\sigma(N / q)}{(N / q)^{1+\varepsilon}} \leq \frac{\sigma(N)}{N^{1+\varepsilon}}
$$

so that

$$
\frac{\sigma(N / q)}{\sigma(N)} \leq \frac{1}{q^{1+\varepsilon}}
$$

Since $\log \log N$ and $\log \log (N / q)$ are positive, it follows that

(28) $\frac{G(N / q)}{G(N)} \leq \frac{\log \log N}{q^{\varepsilon} \log \log (N / q)}=\frac{(N / q)^{\varepsilon} \log \log N}{N^{\varepsilon} \log \log (N / q)}=\exp (g(N / q)-g(N))$,

where $g(t)$ is defined by (20). By Lemma 6, using (21) to define $t_{0}>e$, we have that $g(t)$ is increasing for $t>t_{0}$. Now from (25) we deduce that

$$
e<t_{0}<\frac{N}{p} \leq \frac{N}{q}<N
$$

and from (28) we get $G(N / q)<G(N)$. This shows that $N$ is GA1.

Theorem 6 Infinitely many $C A$ numbers are GA1.

Proof. Choose a sufficiently large prime $p$ satisfying (17), and set $\varepsilon:=F(p, 1)$ (so that $x=p$, by (8)) ). Let $N$ be the largest CA number of parameter $\varepsilon$ (so that $p$ divides $N$, by (12)). From Lemma 3 part (ii) and (17), we get

$$
\log N \geq(x)=(p)>p+c \sqrt{p} \log \log \log p,
$$


so that

$$
\log (N / p)>p+c \sqrt{p} \log \log \log p-\log p>p+1 .
$$

Using the lower bound $\log (1+t) \geq t /(1+t)$, we get

$$
\begin{aligned}
\varepsilon & =F(p, 1)=\frac{\log \left(1+\frac{1}{p}\right)}{\log p} \geq \frac{1}{(p+1) \log p} \\
& >\frac{1}{(p+1) \log (p+1)}>\frac{1}{\log (N / p) \log \log (N / p)}
\end{aligned}
$$

and Lemma 7 implies $N$ is GA1. Since, by Lemma 4, there are infinitely many primes $p$ satisfying (17), the theorem is proved.

\subsection{CA and not GA1}

To study CA numbers that are not GA1, we need a lemma.

Lemma 8 Given a prime $p \geq 3$, let $N$ be the largest $C A$ number of parameter $\varepsilon:=F(p, 1)$. If

$$
\varepsilon<\frac{1}{\log N \log \log N}
$$

then $N$ is not GA1.

Proof. As $\varepsilon=F(p, 1)$, we have $p^{\varepsilon}=(p+1) / p=\sigma(p) / p$. Hence, by (12), inequality (26) becomes an equality when $q=p$, and so do inequalities (27) and (28). Therefore, with $g$ and $t_{0}$ defined by (20) and (21) as in the proof of Lemma 7, we get that

$$
\frac{G(N / p)}{G(N)}=\exp (g(N / p)-g(N))
$$

and, from Lemma 6, that $g(t)$ is decreasing for $t<t_{0}$. Then (29) implies $N / p<N<t_{0}$, so that $G(N)<G(N / p)$. Thus $N$ is not GA1.

The CA numbers $N$ such that $P(N) \in\{2,3,5,7,11,13,29,59,149\}$ are not GA1. There are two CA numbers such that $P(N)=23$; the larger one is not GA1, while the smaller one is GA1. All other CA numbers satisfying $P(N)<300$ are GA1. (These statements follow by computing all CA numbers $N$ with $P(N)<300$, and calculating those that are GA1-see $\S 7$.)

Theorem 7 Infinitely many $C A$ numbers are not GA1. 
Proof. Choose a sufficiently large prime $p$ satisfying (16), and set $\varepsilon:=F(p, 1)$ (so that, from (8),$x=p$ ). Let $N$ be the largest CA number of parameter $\varepsilon$ (so that, from (12), $p=P(N)$ ). From Lemma 3 part (i) and (16), we get

$$
\log N \leq(p)+c \sqrt{p}<p-c \sqrt{p} \log \log \log p+c \sqrt{p}<p,
$$

and so

$$
\varepsilon=\frac{\log \left(1+\frac{1}{p}\right)}{\log p}<\frac{1}{p \log p}<\frac{1}{\log N \log \log N} .
$$

Then Lemma 8 implies $N$ is not GA1. Since there are infinitely many primes $p$ satisfying (16), the theorem is proved.

\subsection{Odd GA1 numbers}

We show that there are infinitely many odd GA1 numbers, and we compute the smallest one.

Let us denote by $\mathcal{P}_{0}=\{2,3,5,7,11,13,17, \ldots\}$ the set of all primes, and by $\mathcal{P}$ a subset of $\mathcal{P}_{0}$. To $\mathcal{P}$, we attach the set

$$
\mathcal{N}_{\mathcal{P}}:=\{n \geq 1: p \mid n \Longrightarrow p \in \mathcal{P}\}
$$

and the function

$$
\mathcal{P}(x):=\sum_{p \in \mathcal{P}, p \leq x} \log p .
$$

A number $N \in \mathcal{N}_{\mathcal{P}}$ is said to be colossally abundant relative to $\mathcal{P}$ (for short, $C A_{\mathcal{P}}$ ) if there exists $\varepsilon>0$ such that

$$
\frac{\sigma(N)}{N^{1+\varepsilon}} \geq \frac{\sigma(n)}{n^{1+\varepsilon}} \quad\left(n \in \mathcal{N}_{\mathcal{P}}\right)
$$

If $M=\prod_{p \in \mathcal{P}_{0}} p^{\alpha_{p}}$ is an ordinary CA number of parameter $\varepsilon$, then the factor $N=\prod_{p \in \mathcal{P}} p^{\alpha_{p}}$ is $\mathrm{CA}_{\mathcal{P}}$, for the same parameter $\varepsilon$, and all $\mathrm{CA}_{\mathcal{P}}$ numbers can be obtained in this way.

Theorem 8 There exist infinitely many odd GA1 numbers.

Proof. First, we observe that Lemma 7 remains valid if we replace CA with $\mathrm{CA}_{\mathcal{P}}$, for any set $\mathcal{P}$ with at least 2 elements.

We set $\mathcal{P}=\mathcal{P}_{0} \backslash\{2\}$. The proof of Theorem 6 remains essentially valid. We just have to change the lower bound for $\log N$ to

$$
\log N \geq \mathcal{P}(p)=(p)-\log 2
$$


and the inequality $\log (N / p)>p+1$ still holds, so that we may conclude that $N$ is GA1.

The smallest $\mathrm{CA}_{\mathcal{P}_{0} \backslash\{2\}}$ number that is GA1 is

$$
\begin{aligned}
\omega & :=1058462574572984015114271643676625 \\
& =3^{4} \cdot 5^{3} \cdot 7^{2} \cdot 11^{2} \cdot 13 \cdot 17 \cdot 19 \cdot 23 \cdot 29 \cdot 31 \cdot 37 \cdot 41 \cdot 43 \cdot 47 \cdot 53 \cdot 59 \cdot 61 \cdot 67 \cdot 71 \cdot 73 .
\end{aligned}
$$

From our computation (see 97.5$)$, $\omega$ is also the smallest odd GA1 number.

Corollary 2 There exist infinitely many GA1 numbers that are not SA.

Proof. This follows immediately from (5) and Theorem 8 ,

Of course, the proof of Theorem 8 works for any set of primes $\mathcal{P}$ such that $\mathcal{P}_{0} \backslash \mathcal{P}$ is finite.

\section{Prime factors of GA1 numbers}

Here we study prime factors of proper GA1 numbers.

\subsection{An upper bound}

We need the following upper bound.

Theorem 9 Given a GA1 number $N$ with $\Omega(N) \geq 3$, let $p$ be a prime factor of $N$. Then for any positive integer $r \leq v_{p}(N)$ we have

$$
p \leq(r \log N)^{1 / r} \leq \log N
$$

Proof. We have $G(N / p) \leq G(N)$, which implies

$$
\frac{\sigma(N / p) N}{(N / p) \sigma(N)} \leq \frac{\log \log (N / p)}{\log \log N}=\frac{\log (\log N-\log p)}{\log \log N}
$$

Note that $\log \log N>\log \log (N / p) \geq \log \log 4>0$. We also have

$$
\begin{aligned}
\log (\log N-\log p) & =\log \left(\log N\left(1-\frac{\log p}{\log N}\right)\right) \\
& =\log \log N+\log \left(1-\frac{\log p}{\log N}\right)
\end{aligned}
$$


so that

$$
\frac{\log (\log N-\log p)}{\log \log N}=1-\frac{-\log \left(1-\frac{\log p}{\log N}\right)}{\log \log N} .
$$

Further, setting $v=v_{p}(N)$, the left side of (30) can be written as

$$
\begin{aligned}
\frac{\sigma(N / p) N}{(N / p) \sigma(N)} & =p \frac{1+p+\cdots+p^{v-1}}{1+p+\cdots+p^{v}} \\
& =1-\frac{1}{1+p+\cdots+p^{v}} \geq 1-\frac{1}{1+p+\cdots+p^{r}} .
\end{aligned}
$$

From (30), (31), and (32), one deduces

$$
p^{r} \leq 1+p+\cdots+p^{r} \leq \frac{\log \log N}{-\log \left(1-\frac{\log p}{\log N}\right)} \leq \frac{\log N \log \log N}{\log p}
$$

which yields

$$
p^{r} \log p \leq \log N \log \log N .
$$

Let us assume, ab absurdum, that $p>(r \log N)^{1 / r}$. Then we would have $p^{r} \log p>(r \log N) \frac{1}{r} \log (r \log N)=\log N \log (r \log N) \geq \log N \log \log N$

contradicting (34). Therefore, $p \leq(r \log N)^{1 / r}$ holds. Finally, by calculus, $(r \log N)^{1 / r}$ is decreasing for $r \geq 1$ (because $\Omega(N) \geq 3$ implies $N \geq 8$ and $\log N>2$ ) and the theorem follows.

\subsection{Study of $\Omega(N)$ where $N$ is GA1}

We show that there are only finitely many proper GA1 numbers $N$ that have a fixed value of $\Omega(N)$.

Theorem 10 If $k \geq 3$, then

$$
\Pi_{k}:=\#\{N: N \text { is } G A 1 \text { and } \Omega(N)=k\}<\infty .
$$

Proof. For a GA1 number $N$ with $\Omega(N)=k>2$, let us write $N=p_{1} p_{2} \cdots p_{k}$ with $p_{1} \leq p_{2} \leq \cdots \leq p_{k}$. We have $N \leq p_{k}^{k}$, so that $p_{k} \geq N^{1 / k}$ holds. But Theorem 9 yields $p_{k} \leq \log N$, whence

$$
\frac{\log N}{\log \log N} \leq k
$$


and $N$ is bounded. Thus $\Pi_{k}$ is finite.

Since $\frac{\log 10^{60}}{\log \log 10^{60}}=28.03 \ldots$, a table of GA1 numbers up to $10^{60}$ (see 97 ) allows us to calculate $\Pi_{k}$ for $k \leq 28$.

We have $\Pi_{k}=0$ if $3 \leq k \leq 12$, and the following table gives $\Pi_{k}$ when $13 \leq k \leq 28$ (see [17, Sequence A201558]).

\begin{tabular}{r|cccccccccccccccc}
\hline$k=$ & 13 & 14 & 15 & 16 & 17 & 18 & 19 & 20 & 21 & 22 & 23 & 24 & 25 & 26 & 27 & 28 \\
$\Pi_{k}=$ & 2 & 4 & 2 & 1 & 1 & 2 & 4 & 1 & 2 & 3 & 7 & 7 & 7 & 1 & 4 & 7 \\
\hline
\end{tabular}

\subsection{The exponent of the largest prime factor}

First, we observe that the function $t \mapsto 2^{t} / t$ is an increasing bijection of the interval $[2,+\infty)$ to itself. Let us introduce the inverse function $h$ defined for $x \geq 2$ by

$$
h(x)=t \quad \Longleftrightarrow \quad x=\frac{2^{t}}{t} .
$$

We shall need the following lemma.

Lemma 9 Let $x$ satisfy $x \geq 2$. Then we have $2 \leq h(x) \leq 3.08 \log x$.

Proof. The lower bound results from the definition of $h$. Let us set $t=h(x)$, so that $x=2^{t} / t$. By noting that $(\log t) / t \leq 1 / e$ holds, we get

$$
\frac{h(x)}{\log x}=\frac{t}{t \log 2-\log t}=\frac{1}{\log 2-(\log t) / t} \leq \frac{1}{\log 2-1 / e}=3.0743 \ldots
$$

which proves Lemma 9.

Theorem 11 Let $N$ be a GA1 number with $\Omega(N) \geq 3$. Set $R=h(\log N)$, so that $2^{R} / R=\log N$. Then $N$ divides the number $M=M(N)$ defined by

$$
M:=\prod_{r=1}^{\lfloor R\rfloor} \prod_{((r+1) \log N)^{1 /(r+1)}<p \leq(r \log N)^{1 / r}} p^{r}=\prod_{r=1}^{\lfloor R\rfloor} \prod_{p \leq(r \log N)^{1 / r}} p .
$$

Proof. Since the function $r \mapsto(r \log N)^{1 / r}$ is decreasing, this follows from Theorem 9 .

For example, if $N=\nu=183783600$, we compute $R=h(\log \nu)=7.072 \ldots$ and find that $M=72 \nu$. 
Theorem 11 allows the computation of proper GA1 numbers - see $\S 7.2$ and $\S 7.5$.

For the exponent $v_{p}(N)$ of a prime $p$ in the standard factorization of $N$, Theorem 11 provides the upper bound $v_{p}(N) \leq v_{p}(M)$, which only depends on the size of $N$.

We now study the exponent of the largest prime factor of a GA1 number.

Theorem 12 Let $N$ be a GA1 number with $\Omega(N) \geq 3$, and let $p=P(N)$ be its largest prime factor. Then $v_{p}(N)=1$.

Proof. Suppose on the contrary that $v:=v_{p}(N) \geq 2$. Then Theorem 9 implies that $N$ divides the number

$$
M_{v}=M_{v}(N):=\left(\prod_{p \leq(v \log N)^{1 / v}} p\right)^{v} \prod_{r=v+1}^{\lfloor R\rfloor} \prod_{p \leq(r \log N)^{1 / r}} p
$$

with $R$ defined by $2^{R} / R=\log N$. Thus, from the function $r \mapsto(r \log N)^{1 / r}$ being decreasing,

$$
\begin{aligned}
\log N & \left.\leq \log M_{v}=v(v \log N)^{1 / v}\right)+\sum_{r=v+1}^{\lfloor R\rfloor}\left((r \log N)^{1 / r}\right) \\
& \leq 2\left((2 \log N)^{1 / 2}\right)+R\left((3 \log N)^{1 / 3}\right) .
\end{aligned}
$$

From Lemmas 9 and 5 , it follows that

$$
\begin{aligned}
\log N & \leq 1.000028\left(2 \sqrt{2 \log N}+3.08 \log \log N(3 \log N)^{1 / 3}\right) \\
& \leq 2.83 \sqrt{\log N}+4.45 \log \log N(\log N)^{1 / 3}
\end{aligned}
$$

\begin{tabular}{|c|c|c|c|c|c|}
\hline$v=$ & 2 & 3 & 4 & 5 & 6 \\
\hline$\left(v \log N_{0}\right)^{1 / v}=$ & 5.48 & 3.56 & 2.78 & 2.37 & 2.12 \\
\hline$M_{v}\left(N_{0}\right)=$ & $43200=2^{6} 3^{3} 5^{2}$ & $1728=2^{6} 3^{3}$ & $64=2^{6}$ & 64 & 64 \\
\hline
\end{tabular}

Therefore, we have

$$
\frac{2.83}{\sqrt{\log N}}+\frac{4.45}{(\log N)^{2 / 3} \log \log N} \geq 1
$$

which implies $\log N \leq 15.03, N \leq N_{0}:=3336369$ and $R \leq 6.65$, so that $N$ must divide $M_{v}\left(N_{0}\right)$ for some $v$ in the range $2 \leq v \leq 6$. But the table

shows that if $v \geq 2$, then the number $M_{v}\left(N_{0}\right)$ divides $M_{2}\left(N_{0}\right)=43200$, contradicting the easily-checked fact that none of the 84 divisors of 43200 is a proper GA1 number. (In fact, we will see in 87.5 that there is no proper GA1 number < 183783600.) This proves the theorem. 


\subsection{The largest prime factor of a GA1 number}

For a GA1 number, we now study the largest prime factor itself.

Theorem 13 For $G A 1$ numbers $N$ with $\Omega(N) \geq 3$, the largest prime factor satisfies

$$
P(N) \sim \log N \quad(N \rightarrow \infty)
$$

Proof. Let $N$ be a GA1 number satisfying $\Omega(N) \geq 3$ and let $p:=P(N)$ be its largest prime factor. From Theorem 9, we know that

$$
p \leq \log N
$$

It remains to get a lower bound for $p$. The proof resembles that of Theorem 12 ,

Since $N$ divides $M$ given by (36) and $p=P(N)$, by Lemma 5 we have

$$
\begin{aligned}
\log N & \leq(p)+\sum_{r=2}^{R}\left((r \log N)^{1 / r}\right) \\
& \leq(p)+\left((2 \log N)^{1 / 2}\right)+\mathcal{O}\left(R(\log N)^{1 / 3}\right) \\
& \leq(p)+\mathcal{O}(\sqrt{\log N}) .
\end{aligned}
$$

From the Prime Number Theorem and from (37), we get

$$
(p)=p+\mathcal{O}(p \exp (-c \sqrt{\log p}))=p+\mathcal{O}(\log N \exp (-c \sqrt{\log \log N})) .
$$

Therefore, (38) becomes

$$
\log N \leq p+\mathcal{O}(\log N \exp (-c \sqrt{\log \log N}))
$$

which, together with (37), completes the proof of the theorem.

\section{Computation of GA1 numbers}

In this section we give several versions of an algorithm to compute GA1 numbers.

\subsection{The Gronwall quotient}

We begin with a lemma and a definition. 
Lemma 10 Let $n$ be a positive integer with $\Omega(n) \geq 3$. Let $q$ and $p$ be prime factors of $n$ satisfying $q<p$ and $v_{q}(n) \leq v_{p}(n)$. Then we have

$$
G(n / q)<G(n / p) \text {. }
$$

Proof. We have

$$
\begin{aligned}
\frac{\sigma(n / q) /(n / q)}{\sigma(n) / n} & =q \frac{\sigma(n / q)}{\sigma(n)}=\frac{q+\cdots+q^{v_{q}(n)}}{1+q+\cdots+q^{v_{q}(n)}}=1-\frac{1}{1+q+\cdots+q^{v_{q}(n)}} \\
& \leq 1-\frac{1}{1+p+\cdots+p^{v_{p}(n)}}=\frac{\sigma(n / p) /(n / p)}{\sigma(n) / n}
\end{aligned}
$$

which implies $\frac{\sigma(n / q)}{n / q} \leq \frac{\sigma(n / p)}{n / p}$.

The lemma follows from $\log \log (n / q)>\log \log (n / p)$, since $\Omega(n) \geq 3$ implies $\log \log (n / p) \geq \log \log 4>0$.

We define the Gronwall quotient $Q(n)$ of a composite integer $n$ to be the number

$$
Q(n):=\max _{\substack{p \mid n \\ p \text { prime }}} \frac{G(n / p)}{G(n)}=\max _{\substack{p \mid n \\ p \text { prime }}} \frac{p^{v_{p}(n)+1}-p}{p^{v_{p}(n)+1}-1} \frac{\log \log n}{\log \log (n / p)} .
$$

GA1 numbers $N$ are characterized by $Q(N) \leq 1$. For example, the " $Q(r)$ " column in Table 1 shows that the only GA1 number $r \in \mathcal{R}$ is $r=4$.

Let us introduce a subset $\mathcal{S}(n)$ of the set of the prime divisors of $n$. The elements of $\mathcal{S}(n)$ are defined by induction. The largest prime factor of $n$ is the first element $q_{1}$ of $\mathcal{S}(n)$. Now let us assume that $i \geq 2$ and that the elements $q_{1}, q_{2}, \ldots, q_{i-1} \in \mathcal{S}(n)$ are known.

If, for all primes $p$ that divide $n$ and are smaller than $q_{i-1}$, we have $v_{p}(n) \leq v_{q_{i-1}}(n)$, then there are no further elements of $\mathcal{S}(n)$, and we get $\mathcal{S}(n)=\left\{q_{1}, q_{2}, \ldots, q_{i-1}\right\}$.

If not, then the element $q_{i} \in \mathcal{S}(n)$ is defined as the largest prime factor of $n$ that satisfies $q_{i}<q_{i-1}$ and $v_{q_{i}}(n)>v_{q_{i-1}}(n)$.

From Lemma 10, if $\Omega(n) \geq 3$ we get

$$
Q(n)=\max _{p \in \mathcal{S}(n)} \frac{G(n / p)}{G(n)}=\max _{p \in \mathcal{S}(n)} \frac{p^{v_{p}(n)+1}-p}{p^{v_{p}(n)+1}-1} \frac{\log \log n}{\log \log (n / p)} .
$$




\subsection{A first algorithm}

To compute all proper GA1 numbers $N \leq x$ for a given $x$, we first calculate $M=M(x)$, defined by

$$
M:=\prod_{r=1}^{\lfloor R\rfloor} \prod_{p \leq(r \log x)^{1 / r}} p
$$

with $R$ such that $(R \log x)^{1 / R}=2$. Any GA1 number $N \leq x$ with $\Omega(N) \geq 3$ is a divisor of $M$ (see Theorem 11).

Thus a first version of the algorithm computes all composite divisors $N$ of $M$, and for each of them calculates $G(N / p) / G(N)$ for all $p \in \mathcal{S}(N)$. If for some $p \in \mathcal{S}(N)$ we have $G(N / p) / G(N)>1$, we stop : $N$ is not GA1. If not, we compute the Gronwall quotient $Q(N)$ (which involves all primes $p$ dividing $N): N$ is GA1 if and only if $Q(N) \leq 1$. 3 .

\subsection{A second algorithm}

A more elaborate version of the algorithm tests only a small number of the divisors of $M$. First, we define

$$
M_{1}:=\prod_{r=2}^{\lfloor R\rfloor} \prod_{p \leq(r \log x)^{1 / r}} p
$$

so that $M_{2}:=M / M_{1}$ is squarefree. Let us write $M_{2}=p_{1} p_{2} \cdots p_{s}$ where $p_{1}, p_{2}, \ldots, p_{s}$ are consecutive primes in ascending order.

As a first step, we compute the set $\mathcal{D}_{0}$ of all the composite divisors of $M_{1}$ and test each of them for GA1 by the method described above.

A divisor of $M$ whose largest prime factor is $p_{i}$ is equal to $d p_{i}$, where $d$ is a divisor of $M$ whose largest prime factor is $<p_{i}$. Therefore, we construct by induction on $i=1,2, \ldots, s$ the set $\mathcal{D}_{i}^{\prime}$ containing those divisors of $M$ whose largest prime factor is $p_{i}$, and the set $\mathcal{D}_{i}$ containing the divisors of $M$ whose largest prime factor is $\leq p_{i}$. Then $\mathcal{D}_{i}^{\prime}$ is equal to $p_{i} \mathcal{D}_{i-1}$ and $\mathcal{D}_{i}=\mathcal{D}_{i}^{\prime} \cup \mathcal{D}_{i-1}$. From Theorem 9, for $i=1,2, \ldots, s$, we only have to test the elements of $\mathcal{D}_{i}^{\prime}$ that are greater than $\exp \left(p_{i}\right)$.

3. To avoid roundoff errors, we carry out our computation in floating point arithmetic with 20 decimal digits and choose a small $\varepsilon$ (typically, $\varepsilon=10^{-5}$ ). In the first step, we keep the $N$ 's satisfying $Q(N) \leq 1+\varepsilon$. For these $N$ 's, we start the computation again with 40 digits. 


\subsection{A third algorithm}

Let us say that a divisor $d \in \mathcal{D}_{i}$ (with $0 \leq i<s$ ) is bad if, for every $j$ satisfying $i<j \leq s$, all multiples of $d$ belonging to $\mathcal{D}_{j}$ are smaller than $\exp \left(p_{j}\right)$.

The largest multiple of $d$ belonging to $\mathcal{D}_{j}$ is $d p_{i+1} p_{i+2} \cdots p_{j}$, so that $d$ is bad if and only if

$$
\log d<\delta_{i}:=\left(p_{i}\right)+\min _{i<j \leq s}\left(p_{j}-\left(p_{j}\right)\right) .
$$

Therefore, we write $\mathcal{G}_{i} \subset \mathcal{D}_{i}$ for the set obtained from $\mathcal{D}_{i}$ by removing the bad divisors, i.e., those divisors $d$ satisfying $d<\Delta_{i}:=\exp \left(\delta_{i}\right)$.

Furthermore, we construct $\mathcal{G}_{i+1}^{\prime}$ and $\mathcal{G}_{i+1}$ by removing from $p_{i+1} \mathcal{G}_{i}$ and $p_{i+1} \mathcal{G}_{i} \cup \mathcal{G}_{i}$, respectively, those divisors $d$ that satisfy $d<\Delta_{i+1}=\exp \left(\delta_{i+1}\right)$.

For $i=1,2, \ldots, s$, it remains to test the elements of $\mathcal{G}_{i}$ whose largest prime factor is equal to $p_{i}$, that is, the elements of $\mathcal{G}_{i}^{\prime}$.

\subsection{Results}

The smallest proper GA1 number is

$$
\nu=183783600=2^{4} \cdot 3^{3} \cdot 5^{2} \cdot 7 \cdot 11 \cdot 13 \cdot 17 .
$$

We compute that $M=M(\nu)=8 \cdot 19 \cdot \nu$ and we find that there is no proper GA1 number $N<\nu$.

Using the third algorithm, we have computed all GA1 numbers $N \leq 10^{60}$ with $\Omega(N) \geq 3$.

These results as well as the Maple code can be found on the web site http://math.univ-lyon1.fr/〜nicolas/GAnumbers.html.

We hope to present soon a fourth algorithm, more sophisticated, and able to compute GA1 numbers up to $10^{120}$.

\section{The number of GA1 numbers up to $x$}

Let $Q_{1}(x)$ be the number of proper GA1 numbers $N \leq x$. From (36) we know that $Q_{1}(x)$ does not exceed the number $\tau(M)$ of divisors of

$$
M=M(x):=\prod_{r=1}^{\lfloor R\rfloor} \prod_{p \leq(r \log x)^{1 / r}} p
$$


with $(R \log x)^{1 / R}=2$. It is easy to see that $\log M \sim \log x$ as $x \rightarrow \infty$, and from the estimation of the large values of the function $\tau$ (cf. [8] or [1] ), it follows that

$$
Q_{1}(x) \leq \exp \left(c \frac{\log x}{\log \log x}\right)
$$

for some positive $c$. By estimating the number of good divisors of $M$ (that is, divisors that are not bad-see $\$ 7.4)$, it might be possible to improve the above estimate.

It seems more difficult to get a lower bound for $Q_{1}(x)$. We hope to return to these questions in another article.

Acknowledgement The authors thank Kieren MacMillan for his help with TeXing the manuscript.

\section{References}

[1] Alaoglu, L., Erdôs, P. : On highly composite and similar numbers. Trans. Amer. Math. Soc. 56, 448-469 (1944)

[2] Briggs, K. : Abundant numbers and the Riemann hypothesis. Experiment. Math. 15, 251-256 (2006). http://www . expmath.org/expmath/volumes/15/15.2/Briggs.pdf (2006). Accessed 23 October 2011

[3] Caveney, G., Nicolas, J.-L., Sondow, J. : Robin's theorem, primes, and a new elementary reformulation of the Riemann Hypothesis. Integers 11, A33. http://www. integers-ejcnt.org/133/133.pdf (2011). Accessed 23 October 2011

[4] Choie, Y.-J., Lichiardopol, N., Moree, P., Sole, P. : On Robin's criterion for the Riemann Hypothesis. J. Théor. Nombres Bordeaux 19, 351366 (2007). http://arxiv.org/abs/math/0604314 (2006). Accessed 23 October 2011

[5] Dusart, P. : Estimates of some functions over primes without R.H. http://arxiv.org/abs/1002.0442v1 (2010). Accessed 23 October 2011

[6] Erdôs, P., Nicolas, J.-L. : Répartition des nombres superabondants. Bull. Soc. Math. Fr. 103, 65-90 (1975). http://www.numdam.org/item?id=BSMF_1975__103__65_0 (1975). Accessed 23 October 2011

[7] Gronwall, T. H. : Some asymptotic expressions in the theory of numbers. Trans. Amer. Math. Soc. 14, 113-122 (1913) 
[8] Hardy, G. H., Wright, E. M. : An Introduction to the Theory of Numbers. Heath-Brown, D. R., Silverman, J. H. (eds.), 6th ed. Oxford University Press, Oxford (2008)

[9] Lagarias, J. C. : An elementary problem equivalent to the Riemann hypothesis. Amer. Math. Monthly 109, 534-543 (2002)

[10] Littlewood, J. E. : Sur la distribution des nombres premiers. C. R. Acad. Sci. Paris Sér. I Math. 158, 1869-1872 (1914)

[11] Nicolas, J.-L., Robin, G. : Majorations explicites pour le nombre de diviseurs de N. Canad. Math. Bull. 26, 485-492 (1983)

[12] Ramanujan, S. : Highly composite numbers. Proc. London Math. Soc. Série 2 14, 347-400 (1915). Also In : Collected Papers, pp. 78-128. Cambridge University Press, Cambridge (1927)

[13] Ramanujan, S. : Highly composite numbers, annotated and with a foreword by J.-L. Nicolas and G. Robin. Ramanujan J. 1, 119-153 (1997)

[14] Robin, G. : Grandes valeurs de la fonction somme des diviseurs et hypothèse de Riemann. J. Math. Pures Appl. 63, 187-213 (1984)

[15] Robin, G. : Sur l'ordre maximum de la fonction somme des diviseurs. In : Séminaire Delange-Pisot-Poitou Paris 1981-82, pp. 233-242. Birkhäuser, Boston (1983)

[16] Schoenfeld, L. : Sharper bounds for the Chebyshev functions $(x)$ and $\psi(x)$. II. Math. Comput. 30, 337-360 (1976)

[17] Sloane, N. J. A. : The On-Line Encyclopedia of Integer Sequences. http://oeis.org (2011). Accessed 10 December 2011 